\title{
The effects of crocin on the symptoms of depression in subjects with metabolic syndrome
}

\author{
Irandokht Nikbakht Jam ${ }^{1, B-D}$, Amir Hossein Sahebkar ${ }^{2, A, E}$, Saeid Eslami ${ }^{3, A}$, Naghmeh Mokhber, A, \\ Mina Nosrati ${ }^{1, B}$, Mohammad Khademi ${ }^{3}, A, B$, Mojtaba Foroutan-Tanha ${ }^{3, B}$, Majid Ghayour-Mobarhan ${ }^{1, A, D-F}$, \\ Farzin Hadizadeh ${ }^{2, A, E}$, Gordon Ferns ${ }^{5, \mathrm{E}}$, Masoumeh Abbasi 6 , B \\ ${ }^{1}$ Metabolic Research Center, School of Medicine, Mashhad University of Medical Sciences, Iran \\ ${ }^{2}$ Biotechnology Research Center, School of Pharmacy, Mashhad University of Medical Sciences, Iran \\ ${ }^{3}$ Pharmaceutical Research Center, School of Pharmacy, Mashhad University of Medical Sciences, Iran \\ ${ }^{4}$ Psychiatry and Behavioral Sciences Research Center, School of Medicine, Mashhad University of Medical Sciences, Iran \\ ${ }^{5}$ Brighton \& Sussex Medical School, Division of Medical Education, Mayfield House, University of Brighton, Staffordshire, UK \\ ${ }^{6}$ Qaem Hospital Cardiology Department, School of Medicine, Mashhad University of Medical Sciences, Iran \\ A - research concept and design; $\mathrm{B}$ - collection and/or assembly of data; $\mathrm{C}$ - data analysis and interpretation; \\ $D$ - writing the article; $E$ - critical revision of the article; $F$ - final approval of article
}

Address for correspondence

Majid Ghayour-Mobarhan

E-mail: GhayourM@mums.ac.ir

Farzin Hadizadeh

E-mail: HadizadehF@mums.ac.ir

Funding sources

None declared

Conflict of interest

None declared

\section{Acknowledgements}

The authors thank the subjects who participated in this study. This work was supported by Mashhad University of Medical Science (MUMS), Iran.

Received on May 21, 2015

Revised on December 15, 2015

Accepted on April 26, 2016

DOI

10.17219/acem/62891

Copyright

Copyright by Author(s)

This is an article distributed under the terms of the

Creative Commons Attribution Non-Commercial License

(http://creativecommons.org/licenses/by-nc-nd/4.0/)

\begin{abstract}
Background. Studies have suggested that metabolic syndrome (MetS) is associated with increased depressive symptoms, and reducing depression in subjects with MetS is important. Crocin, an active component of saffron, has useful properties for subjects with MetS, including antidepressant properties.

Objectives. The aim of the study was to assess the effect of a preparation of crocin on the symptoms of depression in subjects with MetS, and the relationship between changes in those symptoms and the serum pro-oxidant/anti-oxidant balance (PAB).

Material and methods. This sub-study was carried out on 34 subjects with MetS from the authors' previous randomized double-blind controlled clinical trial (RCT), all of whom met the inclusion criteria for this study. The subjects were randomly assigned to treatment and placebo groups ( $n=17$ in each group) and received each $30 \mathrm{mg}$ of crocin (2 tablets of $15 \mathrm{mg}$ ) or placebo for 8 weeks. Depressive symptoms were assessed using the Beck Depression Inventory (BDI). The BDI questionnaire was completed for each subject at the baseline and at the end of the $8^{\text {th }}$ week of treatment. Blood samples were taken from the subjects before and after the intervention period. Statistical analyses were performed using the SPSS for Windows, v. 16 (SPSS Inc., Chicago, USA)
\end{abstract}

Results. Out of the 34 participants enrolled, 33 completed the trial. The degree of depression decreased significantly in the crocin group $(p=0.005)$, but not in the placebo group $(p>0.05)$, and the difference between the 2 groups was statistically significant $(p=0.013)$. No significant relationship was observed between changes in depression symptoms and changes in the serum PAB $(p>0.05)$.

Conclusions. This study demonstrates that at a dose of $30 \mathrm{mg}$ per day for 8 weeks, crocin reduced the symptoms of depression in subjects with Met $S$ compared to the control group, and this effect was independent of its effect on the serum PAB.

Key words: crocin, metabolic syndrome, depression, saffron 
Metabolic syndrome (MetS) is a common disorder that increases the risk of cardiovascular disease and type 2 diabetes. MetS is defined by a set of cardiovascular risk factors including abdominal obesity, hypertension, hyperglycemia and dyslipidemia (high blood triglycerides, low high-density lipoprotein). ${ }^{1}$ MetS has high prevalence worldwide, and also in Iran. ${ }^{2}$ Some studies show that MetS is associated with an increased risk of depressive symptoms. ${ }^{3,4}$ Epidemiological studies show that the chances of patients with MetS developing depression are high and that there is a positive association between components of the metabolic syndrome, including abdominal obesity, hypertriglyceridemia and low HDL cholesterol, and depression. 5,6 Previous studies have pointed to the importance of early detection and management of depression in patients with MetS. ${ }^{7}$

There is evidence suggesting that a significant multifaceted and possibly reciprocal relationship exists between depression and MetS. ${ }^{7-9}$ First, increased inflammatory cytokines and leptin resistance occur in patients with MetS, which increases their chances of developing depression. ${ }^{5,7}$ Evidence also suggests that some metabolic abnormalities in MetS, such as impaired glucose homeostasis and mitochondrial respiration can contribute to the pathophysiology of depression. ${ }^{10}$ It has also been suggested that possible subclinical vascular damage in Mets can contribute to the development of depressive symptoms. ${ }^{11}$ Also, certain components of MetS, including obesity, may be associated with decreased self-esteem and increase the chances of depression. ${ }^{12}$

At the same time, MetS may be characteristic for subjects with depression leading an unhealthy lifestyle, because their diet often contains large amounts of fats and carbohydrates but only small amounts of vegetables, fruits and whole grains. Also, they do not engage in enough physical activity, and these factors make them predisposed to metabolic syndrome. ${ }^{5,7,13}$ Depression is also associated with an increase in chronic inflammation and insulin resistance, and some neuroendocrine effects that can increase the risk of MetS. ${ }^{7}$

Oxidative stress is an imbalance between the production of pro-oxidants and anti-oxidants, with an excess of pro-oxidants. Studies have confirmed the contribution of oxidative stress in the etiology of both depression and MetS. ${ }^{14,15}$ Therefore, reducing oxidative stress could be one of the important goals in the treatment of both MetS and depression. The pro-oxidant/anti-oxidant balance (PAB) assay is a new and simple method to measure the balance of pro-oxidants and anti-oxidants concurrently. Evidence has shown that PAB results are comparable with approved standard assessments of oxidative stress and that they correlate with protein oxidation markers such as carbonyl, advanced glycation end products (AGEs) and advanced oxidative protein products (AOPPs). ${ }^{16,17}$

Previous studies have provided evidence that saffron can be effective in improving depressive symptoms. ${ }^{18}$
Crocin is one of main active components of saffron, and animal studies have confirmed its antidepressant properties. ${ }^{19}$ In addition, previous studies have shown that crocin has anti-oxidant properties as well. ${ }^{19}$

One of crocin antidepressant mechanisms may be related to its anti-oxidant property. The first objective of this study was to evaluate the effects of crocin on the symptoms of depression in subjects with metabolic syndrome. The second objective was to assess the association between changes in the symptoms of depression and the serum PAB, in order to evaluate the relationship between the anti-oxidative and antidepressant effects of crocin. As far as the authors are aware, there has been no previous research similar to this study, and the are no reports in the literature yet on the effects of crocin on depressive symptoms in humans.

\section{Material and methods}

This study is a substudy of the authors' previous randomized double-blind controlled clinical trial (RCT) on subjects with Mets, conducted at the Nutrition Clinic of Qaem Teaching Hospital, Mashhad, Iran, from April to June $2014 .^{20}$ The clinical trial was an approved and registered RCT, compliant with ethical standards, and written consent was obtained from all the participants. Volunteers with MetS according to the International Diabetes Federation (IDF) criteria were randomly assigned to a treatment group and a placebo group, and received either $30 \mathrm{mg}$ of crocin or a placebo for 8 weeks. All subjects received similar dietary advice based on the American Heart Association (AHA) guidelines. This substudy involved 34 of the eligible RCT subjects (Fig. 1).

Exclusion criteria included pregnancy, lactation, age under 18 or over 70 years, use of antidepressant drugs, under 10 points on the Beck Depression Inventory (BDI) questionnaire, grief or unpleasant event during the previous 6 months, and a lack of compliancy in taking the pills regularly.

Fig. 1. Flow chart of the eligibility of study participants

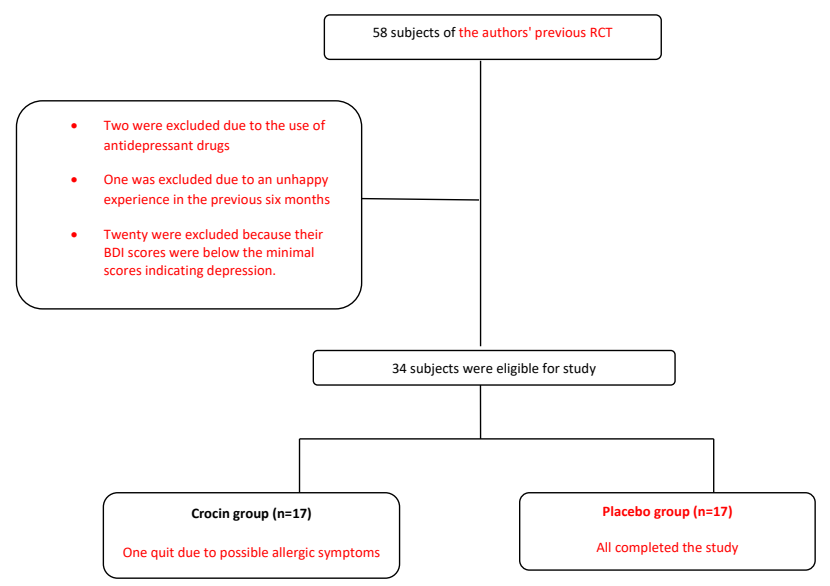


The scores of less than 9 points on the BDI scale indicate a minimal degree of depressive symptoms. Since a drug shows its therapeutic potential in subjects that suffer from a complication rather than in non-affected subjects, this study (like the previous one) included subjects whose BDI score was 10 points or higher. ${ }^{21}$

\section{Symptoms of depression}

The BDI, which was used to assess depressive symptoms, includes 21 questions with 4 options for each question. ${ }^{22}$ The responses to the questions are scored from 0 to 3 according to their intensity, and the total score is used to estimate the degree of depression. The cut-off values of BDI scores to define 4 degrees of depression are 0-9 (grade 1: minimal depression), 10-18 (grade 2: mild depression), 19-29 (grade 3: moderate depression) and 30-63 (grade 4: severe depression). The BDI questionnaire was completed for each subject at the baseline and at the end of week 8 of the study.

\section{Crocin tablets}

The extraction of crocin from saffron was done according to a previously published work by the current authors, and the pill manufacturing was undertaken by the School of Pharmacy, Mashhad University of Medical Science (MUMS), Iran. ${ }^{23}$ Each crocin tablet contained $15 \mathrm{mg}$ of crocin; the placebo pills were given a similar appearance, but contained only starch and food coloring. Quality control tests were conducted under the supervision of an industrial pharmacy specialist. ${ }^{20}$

\section{Anthropometric and blood pressure assessment}

Anthropometric measurements performed on all the participants included height, weight, waist circumference and hip circumference. Standing height $(\mathrm{cm})$ was measured without shoes, with a wall-mounted stadiometer. Maximum hip circumference and minimum waist circumference were measured with a tape to the nearest $0.1 \mathrm{~cm}$. A BC-418 bioelectrical impedance analysis device (Tanita, Tokyo, Japan) was used to measure body weight and body mass index (BMI). Blood pressure was measured on the right arm in the sitting position after at least 15 min of rest.

\section{Blood sampling and laboratory tests}

Blood samples were taken in the morning after $12 \mathrm{~h}$ of fasting, before and after the intervention. After centrifugation, the separated serum was stored at $-80^{\circ} \mathrm{C}$ until the tests were run. Fasting blood lipid profile and blood glucose (FBG) were measured to diagnose metabolic syndrome; the fasting lipid profile included total cholesterol, triglycerides, low-density lipoprotein (LDL) and high-density lipoprotein (HDL). These blood factors were measured enzymatically according to standard protocols and using the relevant commercial kits (Pars Azmoon, Tehran, Iran). The serum PAB measurement was done according to the method described in previous studies. ${ }^{16,17}$

\section{Statistical analysis}

Statistical analyses were performed using the SPSS for Windows v. 16 software package (SPSS Inc., Chicago, USA). The data was analyzed using the KolmogorovSmirnov test to assess normality; Student's t-test or the Mann-Whitney test were used to compare baseline characteristics; the $\chi^{2}$ test, Wilcoxon and Mann-Whitney tests were used to assess depression. A 2-tailed p-value $<0.05$ was considered statistically significant.

\section{Results}

No significant differences were observed in the baseline characteristics of the drug group and the placebo group (Table 1). Similarly, no significant differences were observed in the baseline degree of depression between the 2 groups before the intervention (Table 1 ).

Out of the original 34 volunteers, 33 subjects completed the trial. One participant was excluded due to potential allergic symptoms.

The results of the analyses show that the degree of depression decreased in $68.8 \%$ of the crocin group and $29.4 \%$ of the placebo group (Fig. 2). The reduction of depression in the crocin group was statistically significant, while the reduction in the placebo group was not statistically significant. The difference in the changes in the degree of depression between the 2 groups was statistically significant at the 0.05 level (Table 2).

Fig. 2. Changes in the degree of depression in the 2 groups

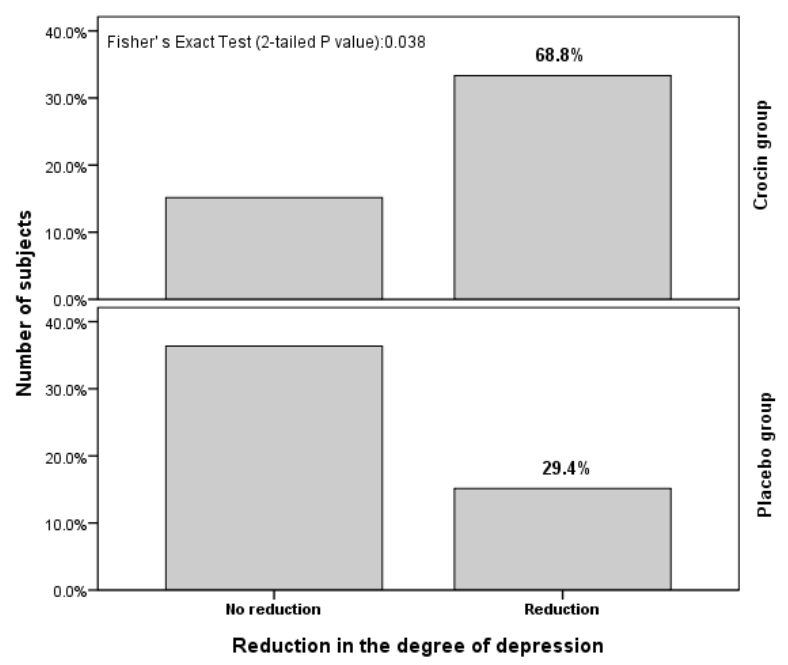


Table 1. Baseline characteristics of the study participants

\begin{tabular}{|c|c|c|c|}
\hline Features & Case group & Control group & ${ }^{*}$ p-value \\
\hline Female/male (n) & $12 / 4$ & $10 / 7$ & $p>0.05$ \\
\hline Age (years) & $45(34.5,50)$ & $48(37,55)$ & $p>0.05$ \\
\hline Weight (kg) & $83.4(79.2,102.2)$ & $85.9(79.3,102.2)$ & $p>0.05$ \\
\hline Height (cm) & $159.5(153.5,166.2)$ & $156(153,167)$ & $p>0.05$ \\
\hline WC (cm) & $110.7(100.7,120.5)$ & $107(102,122.5)$ & $p>0.05$ \\
\hline $\mathrm{HC}(\mathrm{cm})$ & $113.0(112.0,121.7)$ & $113.2(108.5,124.5)$ & $p>0.05$ \\
\hline $\mathrm{BMI}$ & $34.25(31.27,36.2)$ & $34.6(31,38.3)$ & $p>0.05$ \\
\hline Smokers \% (n) & $6.2 \%(1)$ & $0 \%(0)$ & $p>0.05$ \\
\hline Diabetics** \% (n) & $18.7 \%(3)$ & $23.5 \%(4)$ & $p>0.05$ \\
\hline Hypertensive ${ }^{* * *} \%(n)$ & $18.7 \%(3)$ & $18.7 \%(3)$ & $p>0.05$ \\
\hline Cardiovascular disease ${ }^{* * * *} \%(\mathrm{n})$ & $6.2 \%(1)$ & $0 \%(0)$ & $p>0.05$ \\
\hline Degree of depression***** & n (\%) & $\mathrm{n}(\%)$ & ${ }^{*} p$-value \\
\hline Degree 2 (scores 10-18) & $8(50 \%)$ & $10(58 \%)$ & \multirow{4}{*}{$p>0.05$} \\
\hline Degree 3 (scores 19-29) & $5(31.2 \%)$ & $6(35.2 \%)$ & \\
\hline Degree 4 (scores 30-63) & $3(18.7 \%)$ & $1(5.8 \%)$ & \\
\hline BDI Score & $18.5(12.5,26.5)$ & $17(15,22)$ & \\
\hline
\end{tabular}

WC - waist circumference; HC - hip circumference; HDL-C - high-density lipoprotein cholesterol; LDL-C - low-density lipoprotein cholesterol; FBS - fasting blood glucose; DBP - diastolic blood pressure; SBP - systolic blood pressure; BMI - body mass index. Values are expressed as number, median (Q1, Q3) and percentage. *According to the non-normal distribution of data with the Kolmogorov-Smirnov test, non-parametric tests were used. **Diabetes was diagnosed from the medical history, treatment with diabetic drugs or high blood sugar (FBS $\geq 126)$. ***Hypertension was diagnosed from the medical history and treatment with blood pressure drugs. ${ }^{* * *}$ Heart disease was diagnosed from the medical history and treatment with heart disease medications. *****Based on the results of the Beck Depression Inventory (BDI).

BDI scores were significantly reduced in the crocin group ( $p=0.009$ ) but not in the placebo group ( $p>0.05)$. However, the difference between the 2 groups in terms of the magnitude of the changes in BDI scores did not reach statistical significance $(\mathrm{p}=0.09)$.

There was no significant association between changes in serum PAB values and the severity of depression. The magnitude of changes in PAB values was comparable between the subgroups with and without improvement in their BDI scores in both the crocin group and the placebo group $(\mathrm{p}>0.05)$ (Table 3$)$.

\section{Discussion}

The results of the present study showed that at a dose of $30 \mathrm{mg}$ per day for 8 weeks, crocin was associated with a reduction in the degree of depressive symptoms in subjects with MetS. But no association was found between the reduction in the degree of depression and the reduction of serum PAB.

Some previous animal studies have shown that saffron had a much greater antidepressant effect than a placebo; a 2004 study by Hosseinzadeh et al. found that this effect occurs through inhibition of the uptake of dopamine, norepinephrine and serotonin by safranal and crocin, components of saffron. ${ }^{19,24}$ Also, some human clinical trials have compared the antidepressant effect of saffron on mice with standard medications, including imipramine and fluoxetine, and found that saffron can be as effective as standard medications in treating depression. ${ }^{25,26}$ In 2010 Wang et al. found that the antidepressant effect of extract of stigmas of saffron on mice is associated with the presence of crocin. ${ }^{27}$

As noted earlier, studies have confirmed that MetS is associated with depression and that oxidative stress is implicated in the etiology of both depression and metabolic syndrome. $^{14,15}$ The antidepressant mechanisms of saffron and its components are not still clear. In vitro and animal studies have shown the effectiveness of crocin in reducing depression, as well as its anti-oxidant properties. ${ }^{19}$ Therefore, crocin may be effective in treating both depression and oxidative stress in patients with Mets.

As far as the authors are aware, no study has previously been published about the effect of crocin on depression in human subjects. In 2011 Shemshian et al. conducted a study involving saffron and the BDI questionnaire. ${ }^{21}$ They assessed the effect of 100 mg of saffron per day for 12 weeks, comparing it with a placebo on 60 patients with depression and anxiety. They found that saffron significantly reduced the BDI scores of depression in the patients, and concluded that saffron has a therapeutic antidepressant effect on patients with a clinical diagnosis of depression. The present study, on the other hand, assessed the effect of crocin on the symptoms of depression in subjects with MetS who have a mild to moderate degree of depression according to the BDI scale. Shemshian et al. used $100 \mathrm{mg}$ saffron per day and the present study used $30 \mathrm{mg}$ of crocin per day. Crocin comprises $10 \%$ of the dry weight of saffron, so the crocin dose in the present study was 3 times higher than in their study. However, in the study by Shemshian et al. there were other active components of saffron in addition to crocin.

The present study found no association between the reduction in the degree of depression and the serum PAB. This means that the results of this study did not support the theory that anti-oxidant action of crocin is involved in reducing the symptoms of depression in subjects with 
Table 2. Comparison of changes in the degree of depression in the 2 groups

\begin{tabular}{|c|c|c|c|c|}
\hline $\begin{array}{l}\text { Study group } \\
\text { and statistical test }\end{array}$ & $\begin{array}{c}\text { Degree of } \\
\text { depression* } \\
\text { median (Q1-Q3) } \\
\text { Week } 0\end{array}$ & $\begin{array}{c}\text { Degree of } \\
\text { depression* } \\
\text { median (Q1-Q3) } \\
\text { Week } 8\end{array}$ & $\begin{array}{l}\text { Reduction in } \\
\text { the degree of } \\
\text { depression } n(\%)\end{array}$ & $\begin{array}{c}\text { Wilcoxon test } \\
\text { (2-tailed p-value) }\end{array}$ \\
\hline Crocin group $(n=16)$ & $2.5(2-3)$ & $2(1-2.75)$ & $11(68.8 \%)$ & $p=0.005$ \\
\hline Placebo group $(n=17)$ & $2(2-3)$ & $2(2-3)$ & $5(29.4 \%)$ & $p>0.05$ \\
\hline $\begin{array}{l}\text { Mann-Whitney test for } \\
\text { changes in the degree of } \\
\text { depression in the } 2 \text { groups }\end{array}$ & \multicolumn{4}{|c|}{$p=0.013$ (2-tailed p-value) } \\
\hline
\end{tabular}

*The degree of depression based on the results of the Beck Depression Inventory (BDI) as follows: degree 1 - minimal depression (scores 0-9); degree 2 - mild depression (scores 10-18); degree 3 - moderate depression (scores 19-29) and degree 4 - severe depression (scores 30-63).

Table 3. Serum PAB changes in relation to reductions in the degree of depression

\begin{tabular}{|c|c|c|}
\hline The study groups & $\begin{array}{l}\text { Reduction in the degree of } \\
\text { depression ( } \mathrm{n} \%)\end{array}$ & $\begin{array}{l}\text { PAB changes median } \\
(\text { Q1, Q3) }\end{array}$ \\
\hline \multirow{2}{*}{ Crocin group $(n=16)$} & $\begin{array}{c}\text { reduction } \\
(n=11,68.8 \%)\end{array}$ & $-17.7(-51.9,5.2)$ \\
\hline & $\begin{array}{l}\text { no reduction } \\
(n=5,31.2 \%)\end{array}$ & $-4.9(-22.31,8.98)$ \\
\hline \multirow{2}{*}{ Placebo group $(n=17)$} & $\begin{array}{l}\text { reduction } \\
(\mathrm{n}=5,29.4 \%)\end{array}$ & $-15.9(-23.9,9.6)$ \\
\hline & $\begin{array}{l}\text { no reduction } \\
(n=12,70.6 \%)\end{array}$ & $-6.82(-34.3,35.7)$ \\
\hline $\begin{array}{l}\text { Mann-Whitney test for the relation between changes } \\
\text { in depression and } \mathrm{PAB}^{+}\end{array}$ & \multicolumn{2}{|c|}{$\mathrm{ns}^{*}$} \\
\hline $\begin{array}{l}\text { Spearman correlation for changes in depression and } \\
\text { PAB in the } 2 \text { groups }\end{array}$ & \multicolumn{2}{|c|}{$\mathrm{ns}^{*}$} \\
\hline
\end{tabular}

${ }^{*}$ ns - not significant $(p>0.05) ;{ }^{\dagger}$ In this Mann-Whitney test, reduction in the degree of depression (as two variables: 1 - no reduction, 2 - reduction) was the grouping variable, and change in serum PAB was the test variable.

MetS. It is likely that the main antidepressant mechanism of crocin is preventing the uptake of the neurotransmitters such as dopamine, norepinephrine and serotonin, as previous evidence has suggested, but this needs to be further explored in future clinical studies.

The main limitation of this study is that it included subjects who did not have a clinical diagnosis of depression, because it was a substudy of research that was not primarily designed to assess the impact of crocin on the participants' psychological status. ${ }^{20}$ Other limitations include the small sample size, the evaluation of the effects of only 1 dosage ( $30 \mathrm{mg}$ per day), and the relatively short duration of the treatment and follow-up. All of these limitations need to be addressed in future studies.

\section{Conclusions}

The results of this study showed that supplementation with crocin at a daily dose of $30 \mathrm{mg}$ for 8 weeks could reduce the symptoms of depression in subjects with MetS, an effect that appears to be independent of changes in plasma PAB status.

\section{References}

1. Mottillo S, Filion KB, Genest J, et al. The metabolic syndrome and cardiovascular risk a systematic review and meta-analysis. J Am Coll Cardiol. 2010;56(14):1113-1132.

2. Azizi F, Salehi P, Etemadi A, Zahedi-AsI S. Prevalence of metabolic syndrome in an urban population: Tehran lipid and glucose study. Diabetes Res Clin Pract. 2003;61(1):29-37.

3. Sekita A, Arima H, Ninomiya T, et al. Elevated depressive symptoms in metabolic syndrome in a general population of Japanese men: A cross-sectional study. BMC Public Health. 2013;13:862.

4. Foley DL, Morley KI, Madden PA, Heath AC, Whitfield JB, Martin NG. Major depression and the metabolic syndrome. Twin research and human genetics. The Official Journal of the ISTS. 2010;13(4):347-358.

5. Marazziti D, Rutigliano G, Baroni S, Landi P, Dell'Osso L. Metabolic syndrome and major depression. CNS Spectrums. 2014;19(4):293-304.

6. Koponen H, Jokelainen J, Keinanen-Kiukaanniemi S, Kumpusalo E, Vanhala M. Metabolic syndrome predisposes to depressive symptoms: A population-based 7-year follow-up study. J Clin Psychiatry. 2008;69(2):178-182.

7. Pan A, Keum N, Okereke Ol, et al. Bidirectional association between depression and metabolic syndrome: A systematic review and metaanalysis of epidemiological studies. Diabetes Care. 2012;35(5):1171-1180.

8. Goldbacher EM, Bromberger J, Matthews KA. Lifetime history of major depression predicts the development of the metabolic syndrome in middle-aged women. Psychosomatic Medicine. 2009;71(3):266-272.

9. Kemp DE, Ismail-Beigi F, Calabrese JR. Antidepressant response associated with pioglitazone: Support for an overlapping pathophysiology between major depression and metabolic syndrome. Am J Psychiatry. 2009;166(5):619. 
10. Mclntyre RS, Soczynska JK, Konarski JZ, et al. Should depressive syndromes be reclassified as "Metabolic Syndrome Type II"? Ann Clin Psychiatry. Official Journal of the American Academy of Clinical Psychiatrists. 2007;19(4):257-264.

11. Vykoukal D, Davies MG. Vascular biology of metabolic syndrome. Journal of Vascular Surgery. 2011;54(3):819-831.

12. Luppino FS, de Wit LM, Bouvy PF, et al. Overweight, obesity, and depression: A systematic review and meta-analysis of longitudinal studies. Arch Gen Psychiatry. 2010;67(3):220-229.

13. Bonnet F, Irving K, Terra JL, Nony P, Berthezene F, Moulin P. Anxiety and depression are associated with unhealthy lifestyle in patients at risk of cardiovascular disease. Atherosclerosis. 2005;178(2):339344.

14. Sankhla M, Sharma TK, Mathur K, et al. Relationship of oxidative stress with obesity and its role in obesity induced metabolic syndrome. Clinical Laboratory. 2012;58(5-6):385-392.

15. Michel TM, Pulschen D, Thome J. The role of oxidative stress in depressive disorders. Current Pharmaceutical Design. 2012;18(36):5890$-5899$.

16. Alamdari DH, Paletas K, Pegiou T, Sarigianni M, Befani C, Koliakos G. A novel assay for the evaluation of the prooxidant-antioxidant balance, before and after antioxidant vitamin administration in type II diabetes patients. Clin Biochem. 2007;40:248-254.

17. Alamdari $\mathrm{DH}$, Ordoudi $\mathrm{SA}$, Nenadis N, et al. Comparison of prooxidant-antioxidant balance method with crocin method for determination of total prooxidant-antioxidant capacity. Iran J Basic Med Sci. 2009;12:93-99.

18. Hausenblas HA, Saha D, Dubyak PJ, Anton SD. Saffron (Crocus sativus L.) and major depressive disorder: A meta-analysis of randomized clinical trials. Journal of Integrative Medicine. 2013;11(6): 377-383.

19. Alavizadeh $\mathrm{SH}$, Hosseinzadeh $\mathrm{H}$. Bioactivity assessment and toxicity of crocin: A comprehensive review. Food and chemical toxicology. An International Journal Published for the British Industrial Biological Research Association. 2014;64:65-80.

20. Nikbakht-Jam I, Khademi M, Eslami S, et al. Effect of crocin on prooxidant-antioxidant balance in subjects with metabolic syndrome: A randomized, placebo-controlled clinical trial (in press).

21. Shemshian AS, Kermani T, Seyed HM, et al. Saffron (Crocus sativus) in the treatment of anxiety and depression: A double-blind, randomized and placebo-controlled trial. Clin Biochem. 2011;44(13):S118.

22. Beck AT, Ward CH, Mendelson M, Mock J, Erbaugh J. An inventory for measuring depression. Arch Gen Psychiatry. 1961;4:561-571.

23. Hadizadeh F, Mohajeri SA, Seifi M. Extraction and purification of crocin from saffron stigmas employing a simple and efficient crystallization method. Pakistan Journal of Biological Sciences: PJBS. 2010;13(14):691-698.

24. Hosseinzadeh, H, Karimi G, Niapoor M. Antidepressant effects of crocus sativus stigma extracts and its constituents, crocin and safranal, in mice. J Med Plants. 2004;3:48-58.

25. Akhondzadeh S, Fallah-Pour H, Afkham K, Jamshidi AH, KhalighiCigaroudi F. Comparison of Crocus sativus L. and imipramine in the treatment of mild to moderate depression: A pilot double-blind randomized trial. BMC Complement Altern Med. 2004;4:12.

26. Noorbala AA, Akhondzadeh S, Tahmacebi-Pour N, Jamshidi AH. Hydro-alcoholic extract of Crocus sativus L. versus fluoxetine in the treatment of mild to moderate depression: A double-blind, randomized pilot trial. J Ethnopharmacol. 2005;97(2):281-284.

27. Wang $Y$, Han $T$, Zhu $Y$, et al. Antidepressant properties of bioactive fractions from the extract of Crocus sativus L. Journal of Natural Medicines. 2010;64(1):24-30. 